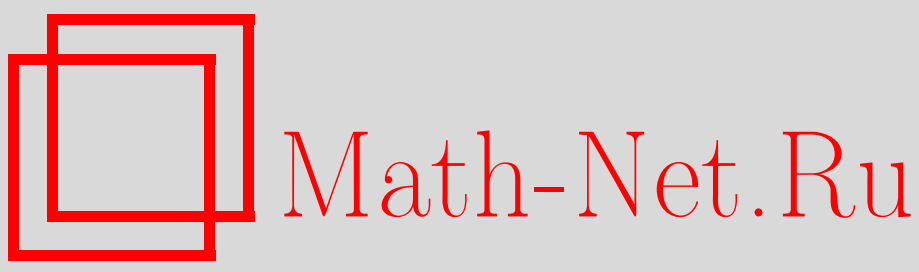

Т. В. Дубровина, Н. И. Дубровин, Конусы в группах, Матем. сб., 1996, том 187, номер 7, 59-74

DOI: https://doi.org/10.4213/sm144

Использование Общероссийского математического портала Math-Net.Ru подразумевает, что вы прочитали и согласны с пользовательским соглашением

http: //www.mathnet.ru/rus/agreement

Параметры загрузки:

IP : 54.84 .234 .179

26 апреля 2023 г., 13:25:35 
УДК 512.8

\author{
Т.В. Дубровина, Н.И. Дубровин
}

\title{
Конусы в группах
}

\begin{abstract}
Изучается структура идеалов конуса $P$ группы $G$. Полностью разбирается случай, когда $P$ имеет один вполне первичный идеал. Строятся соответствующие примеры на основе универсальной накрьвающей группы специальной линейной группы $2 \times 2$-матриц над полем $\mathbb{R}$.

Библиография: 10 названий.
\end{abstract}

\section{Введение}

Подмножество $P$ группы $G$ назовем конусом, если $P \cdot P \subseteq P$ и $P \cup P^{-1}=G$. Если, кроме того, $P \cap P^{-1}=\{1\}$ (здесь и далее 1 - единичньй элемент групшы $G$ ), то $P$ назовем основным конусом группы $G$. В работе изучается структура идеалов конусов, в частности, первичных и вполне первичных идеалов. Существует почти полная аналогия между этой задачей и исследованием структуры идеалов цепных областей в силу следующего легко проверяемого утверждения.

ПРЕДЛОЖЕНИЕ 1. Подкольцо $R$ тела $D$ является чепным порядком в $D$ (т.е. как левые, так и правые идеаль кольца $R$ линейно упорядочены по включению) в том и только том случае, когда полугруппа $\left(R^{*}, \cdot\right)$ ненулевых әлементов кольиа $R$ будет конусом в мультипликативной группе тела $D$.

Для полной аналогии остается положительно ответить на следующий вопрос: для каждого ли основного конуса $P$ групшы $G$ сушествует ассоциированный с ним цепной порядок $R$ в теле $D$; ассоциированный в том смысле, что $(G, \cdot) \subseteq\left(D^{*}, \cdot\right)$, $G \cap R=P$, и любой ненулевой элемент $d \in D$ может быть записан в виде $d=g_{1} u_{1}=u_{2} g_{2}$, где $u_{1}, u_{2}$ обратимы в $R$, а $g_{1}, g_{2} \in G$ таковы, что $P g_{1} P=P g_{2} P$ (см. $[1, \S 1$, определение 4])? Отметим, что этот вопрос включает в себя, в частности, проблему Мальцева о вложимости групповой алгебры правоупорядоченной группы в тело (см. [2, проблема 17, с. 87]), ибо группа $G$ с линейньп порядком $\leqslant$ будет правоупорядоченной в том и только том случае, если множество $P(G)=\{g \in G \mid g \geqslant 1\}$ является основным конусом в $G$; тогда неравенство $g_{1} \leqslant g_{2}$ равносильно принадлежности $g_{2} g_{1}^{-1} \in P(G)$ (см. [2, глава V, $\left.\left.\S 4\right]\right)$.

Другая причина возникшего интереса к конусам заключается в том, что с этим тесно связано изучение автоморфизмов линейно упорядоченных множеств (см. $\S 3$, и именно на этом пути получается полное описание конусов с одним вполне первичньм идеалом (см. примеры в $§ 4$, а также теорему 4).

В работе упоминается пример фундаментальной группы трилистника с основньп конусом, содержащим первичный, но не вполне первичный идеал, коммутант которой не содержится в собственной выпуклой подгруппе (см. [2, проблема 1, с. 34],

(С) Т. В. ДУБровина, Н. И. Дувровин 1996 
а также [3] и [4]). Отметим попутно, что в работе Бергмана [5] приводится пример правоупорядоченной, но не локально-индикабельной группы. Эти примеры объединяет то, что соответствующие группы вложены в один и тот же универсальньй объект - универсальную накрывающую группу группы $\operatorname{SL}(2, \mathbb{R})$.

\section{§1. Основные свойства конусов}

Всюду далее в этом параграфе $P$ - конус в группе $G$. Непустое подмножество $I$ групшы $G$ назовем правым $P$-идеалом, если $I P \subseteq I$ и $I \subseteq g P$ для некоторого $g \in G$. Аналогично определяется понятие левого $P$-идеала. Если $I-$ правый и левый $P$-идеал одновременно, то говорим в этом случае, что $I-P$-идеал. При дополнительном условии $I \subseteq P$ приставку " $P$-" опускаем и называем $I$ (правым, левьм) идеалом. Если, кроме того, $I \neq P$, то (правьй, левый) идеал $I$ назьваем собственным. Собственный идеал $I$ конуса $P$ называется первичнылм (вполне первичным), если из включения $a P b \subseteq I(a b \in I)$ для элементов $a, b \in P$ следует, что либо $a \in P$, либо $b \in P$. Множество первичных идеалов конуса $P$ обозначим spec $P$, а множество вполне первичных идеалов обозначим $\overline{\operatorname{spec}} P$.

ПРЕДЛОЖЕНИЕ 2. Множество правых (левых) Р-идеалов линейно упорядочено по включению.

ПРЕДЛОЖЕНИЕ 3. Множсество обратимых элементов конуса $P$ совпадает с $P \cap P^{-1}$ и является подгруппой группьи $G$.

Это множество далее будем обозначать $U(P)$. В частности, $P$ - основной конус в том и только том случае, когда $U(P)=\{1\}$.

ПРЕДЛОЖЕНИЕ 4. Множество $J(P)$ необратимых в $P$ әлементов будет максимальным правым (левым ) собственным идеалом. Идеал $J(P)$ вполне первичен и имеет место разлохсение

$$
P=U(P) \cup J(P) ; \quad U(P) \cap J(P)=\varnothing .
$$

Идеал $J(P)$ называем далее радикалом конуса $P$.

Доказательства предложений 2-4 непосредственны и аналогичны соответствующим утверждениям о цепных кольцах.

Лемма 1. Пусть $J$-вполне первичный идеал конуса $P$. Тогда $а J=J а=J$ для любого әлемента $a \in P \backslash J$.

ДокАЗАТЕЛЬСтво. Если $b \in J$, то $b P \subseteq a P$ (см. предложение 2); значит, $c=a^{-1} b \in P$. Так как $a c=b \in J$ и $a \notin J$, то $c \in J$ по определению вполне первичного идеала, т.е. $b \in a J$. Итак, доказано, что $J \subseteq a J$. Обратное включение очевидно, поэтому $J=a J$. Аналогично доказывается равенство $J=J a$.

Подмножество $Q$ группы $G$ назовем надконусом конуса $P$, если $Q \cdot Q \subseteq Q$ и $Q \supseteq P$. Ясно, что $Q$ будет конусом в этом случае, и если $Q \neq G$, то $Q$ будет $P$-идеалом.

ТЕоремА 1 (локализация по вполне первичному идеалу). Пусть $J-$ вполне первичный идеал конуса $P$. Тогда множсество $Q=(P \backslash J)^{-1} \cup P$ будет надконусом конуса $P$, радикал которого совпадает $с$ J, а множество обратимых әлементов совпадает $с(P \backslash J)^{-1} \cup(P \backslash J)$. 
ДоКАЗАТЕЛьство. Если $a, b \in P \backslash J$, то $a b \in P \backslash J$ в силу вполне первичности $J$. Следовательно, $b^{-1} a^{-1} \in(P \backslash J)^{-1}$. Далее, если $b P \supseteq a P$, то $b^{-1} a \in P$. В случае $b P \subset a P$ имеем: $b^{-1} a=\left(a^{-1} b\right)^{-1} \in(P \backslash J)^{-1}$, так как $a^{-1} b \in P \backslash J$. Остается рассмотреть произведения вида $a^{-1} p, p a^{-1}$ для $p \in J$. В этом случае $a^{-1} p, p a^{-1} \in J$ в силу леммы 1 . Итак, доказано, что $Q-$ надконус.

Ясно, что $J(Q) \subseteq J$, ибо элементы из множеств $(P \backslash J)^{-1}$ и $P \backslash J$ обратимы в $Q$. Но с другой стороны, $J$ - идеал конуса $Q$, что следует из леммы 1 . Тогда $J(Q)=J$ и $U(Q)=Q \backslash J(Q)=(P \backslash J)^{-1} \cup(P \backslash J)$.

Надконус $Q$, построенный в теореме 1 , назовем локализацией конуса $P$ по вполне первичному идеалу $J$ и будем обозначать $P_{J}$.

СлЕДСТВИЕ. Существует взаимно однозначное и обращающее включение соответствие между множеством вполне первичных идеалов конуса $Р$ и множеством Cоп $P$ надконусов конуса $P$, не совпадающих с $G$. Это соответствие задается правилами:

$$
\begin{aligned}
& J \in \overline{\operatorname{spec}} P \rightarrow P_{J} \in \operatorname{Con} P, \\
& Q \in \operatorname{Con} P \rightarrow J(Q) \in \overline{\operatorname{spec}} P .
\end{aligned}
$$

ДокАЗАтЕЛьство. Ясно, что соответствия (2) и (3) обращают включения. Кроме того, равенство $J\left(P_{J}\right)=J$ доказано в теореме 1 . Наоборот, пусть $J=J(Q)$ для некоторого $Q \in \operatorname{Con} P$. Так как $Q \neq G$, то $J \neq \varnothing$. Кроме того, $J-$ вполне первичный идеал конуса $Q$ (предложение 4 ), а значит, и конуса $P$. Тогда $P_{J}=P \cup(P \backslash J)^{-1} \subseteq Q$ и $(Q \backslash P)^{-1} \subseteq P \backslash J$, откуда $Q \subseteq P \cup(P \backslash J)^{-1}=P_{J}$. Равенство $P_{J}=Q$, а вместе с тем и следствие доказаны.

Пусть $I$ - непустое подмножество групшы $G$. Введем обозначения

$$
\begin{aligned}
O_{r}(I) & =\{g \in G \mid I g \subseteq I\}, \\
O_{l}(I) & =\{g \in G \mid g I \subseteq I\}, \\
I^{-1} & =\{g \in G \mid I g I \subseteq I\},
\end{aligned}
$$

которые и назовем, соответственно, правым множителем, левым множителем и обратным множсеством множества $I$. Отметим, что $O_{r}(I)$ и $O_{l}(I)$ будут подмоноидами группы $G$, а

$$
I^{-1}=\left\{g \in G \mid I g \subseteq O_{l}(I)\right\}=\left\{g \in G \mid g I \subseteq O_{r}(I)\right\} .
$$

Далее нетрудно проверить, что если $I$ - правьй $P$-идеал, то $O_{l}(I)-$ конус, а $O_{r}(I)$ - надконус конуса $P ; I$ является правым $O_{r}(I)$ - и левым $O_{l}(I)$-идеалом, а $I^{-1}$ является правым $O_{l}(I)$ - и левьм $O_{r}(I)$-идеалом. Аналогичное утверждение имеет место и для левых $P$-идеалов.

ТЕорема 2 (характеризация главных односторонних идеалов). Пусть $I-$ односторонний $P$-идеал. Обозначим $S=O_{r}(I), Q=O_{l}(I)$. Тогда либо $I$ - главный правый $S$-идеал и главный левый $Q$-идеал одновременно; либо $I$ и $J(S)$ не являются главными правыми $S$-идеалами, $J(Q)$ и $I$ не являются главными левьлми $Q$-идеалами и $I^{-1} I=J(S), I I^{-1}=J(Q), I=I J(S), J(Q) I=I$.

Если $I-$ правый $P$-идеал и $I J(P) \neq I$, то $S=P$ и $I$ - главный правый P-идеал. 
ДокАЗАТЕЛЬСТво. Предположим, что либо $I^{-1} I=S$, либо $I I^{-1}=Q$. Пусть $I^{-1} I=S$. Тогда $b a=1$ для некоторых элементов $b \in I^{-1}$ и $a \in I$. Следовательно, $a b=1$ и $c=a(b c) \in a S$ для любого элемента $c \in I$. Отсюда вытекает включение $I \subseteq a S$. В силу очевидности обратного включения, получаем равенство $I=a S$. Тогда $Q=a S a^{-1}, I=Q a$, тем самьм имеет место первый случай альтернативы. Аналогичный результат получаем, когда $I I^{-1}=Q$.

Далее понадобятся две леммы.

ЛЕмма 2. Имеют место равенства $O_{l}\left(I^{-1}\right)=S, O_{r}\left(I^{-1}\right)=Q$.

ДокАЗАтЕльство. Предположим, что $O_{l}\left(I^{-1}\right) \supset S$. Тогда $I \neq I O_{l}\left(I^{-1}\right)$, поэтому $I \subset Q c \subseteq I O_{l}\left(I^{-1}\right)$ для любого элемента $c \in I O_{l}\left(I^{-1}\right) \backslash I$. Отсюда вытекает, что $I c^{-1} \subset Q$, а значит, $c^{-1} \in I^{-1}$. Далее имеем: $1=c \cdot c^{-1} \in$ $I O_{l}\left(I^{-1}\right) I^{-1}=I I^{-1}$ и согласно доказанному выше $I=a S$ - главный правый $S$-идеал. Тогда $O_{l}\left(I^{-1}\right)=O_{l}\left(S a^{-1}\right)=S$, что противоречит предположению. Противоречие доказывает справедливость равенства $O_{l}\left(I^{-1}\right)=S$. Аналогично доказывается равенство $O_{r}\left(I^{-1}\right)=Q$.

Лemma 3. $O_{l}(J(S))=O_{r}(J(S))=S$.

ДокАЗАТЕЛЬСТво. Если $a \in G \backslash S$, то $a^{-1} \in J(S)$; поэтому $J(S) a \supset J(S)$, а значит, $a \notin O_{r}(J(S))$.

Продолжим доказательство теоремы. Предположим, что $I \neq I J(S)$. Тогда $I \supseteq a S \supset I J(S) \supseteq a J(S)$ для элемента $a \in I \backslash I J(S)$. Так как между $a S$ и $a J(S)$ нет правых $S$-идеалов, то $I J(S)=a J(S)$. Отсюда $a^{-1} I \subseteq O_{l}(J(S))=S$ (лемма 3 ), что дает включение $I \subseteq a S$ и тем самым равенство $I=a S$. Тогда снова имеет место первый случай альтернативы. Заодно мы доказали последнее утверждение теоремы. Неравенство $I \neq J(Q) I$ аналогично ведет к первому случаю альтернативы.

Предположим теперь, что $I^{-1} I \neq S$ и $I I^{-1} \neq Q$. Если либо $I^{-1} I \neq J(S)$, либо $J(S)$ был бы главным правым $S$-идеалом, то $I^{-1} I \subseteq z S$ для подходящего элемента $z \in J(S)$. Тогда $z^{-1} I^{-1} I \subseteq S$, откуда $z^{-1} I^{-1} \subseteq I^{-1}$, а значит, $z^{-1} \in O_{l}\left(I^{-1}\right)=S$ (лемма 2). Получаем, что $z$ - обратимый в $S$ элемент, принадлежащий радикалу $J(S)$. Это противоречие показывает, что на самом деле $I^{-1} I=J(S)$, и $J(S)$ не является главньм правым $S$-идеалом. Аналогично доказывается равенство $I I^{-1}=J(Q)$ и тот факт, что $J(Q)$ и $I$ не являются главными левыми $Q$-идеалами.

Доказательство теоремы закончено.

СлЕДСТВИЕ. В условиях и обозначениях теоремь 2 , если $I \neq\left(I^{-1}\right)^{-1}$, то $I=z J(S)=J(Q) z, I^{-1}=S z^{-1}=z^{-1} Q u\left(I^{-1}\right)^{-1}=z S=Q z$ для некоторого әлемента $z \in G$.

ДокаЗАтЕльство. Выберем $z \in\left(I^{-1}\right)^{-1} \backslash I$, ибо заведомо $I \subseteq\left(I^{-1}\right)^{-1}$. Тогда, во-первых, $I$ не является главным правым $S$-идеалом, а во-вторых, согласно лемме 2 и теореме 2

$$
S=O_{l}\left(I^{-1}\right) \supseteq I^{-1}\left(I^{-1}\right)^{-1} \supseteq I^{-1} z \supseteq I^{-1} I=J(S) .
$$


Если $I^{-1} z=S$, то $I^{-1}=S z^{-1}, I=z J(S), Q=z S z^{-1}$ и все остальные равенства в формулировке следствия проверяются немедленно. Если же $I^{-1} z \neq S$, то из (4) вытекает, что $I^{-1} z=J(S)$, т.е. $I^{-1}=J(S) z^{-1}$. Отсюда получаем: $J(S)=I^{-1} I=J(S) z^{-1} I$. Тогда $z^{-1} I \subseteq S$ согласно лемме 3 , поэтому $z^{-1} \in I^{-1}$, - противоречие с равенством $I^{-1}=J(S) z^{-1}$. Противоречие показывает, что случая $I^{-1} z \neq S$ быть не может; тем самым следствие доказано.

Теорема 3. Пусть $I$ - правый $P$-идеал с условием $O_{r}(I)=P$. Обозначим $S=O_{l}(I)$. Отображсения

$$
\begin{aligned}
& J \in \operatorname{spec} P \rightarrow I J I^{-1}, \\
& B \in \operatorname{spec} S \rightarrow I B I^{-1}
\end{aligned}
$$

являются взаимно обратными изоморфизмами линейно упорядоченных по включению множеств $\operatorname{spec} P$ u $\operatorname{spec} S$. При этом $P$-идеал $J$ вполне первичен тогда и только тогда, когда $S$-идеал $I J I^{-1}$ вполне первичен.

ДокАЗАТЕЛЬСТво. Если либо $I^{-1} I=P$, либо $I I^{-1}=S$, то $I$ - главный правый $P$-идеал, и справедливость утверждения проверяется непосредственно. В противном случае согласно теореме 2 получим: $I^{-1} I=J(P)$ и $I I^{-1}=J(S)$.

Лемма 4. А. Если $A_{1}$ и $A_{2}$ - левъе $P$-идеалы такие, что $A_{1} \supset A_{2} u$ $I A_{1}=I A_{2}$, mо $A_{1}=P z$ и $A_{2}=J(P) z$ для некоторого $z \in G$.

Б. Если $A_{1}$ и $A_{2}-P$-идеаль такие, что $A_{1} \supset A_{2}$ и $I A_{1} I^{-1}=I A_{2} I^{-1}$, mо либо $A_{1}$ и $A_{2}$ как в $n$. A, либо $A_{1}=z P, A_{2}=z J(P)$ для некоторого $z \in G$.

Доказательство. А. Из условия вытекает, что $J(P) A_{1}=J(P) A_{2} \subseteq A_{2} \subset$ $A_{1}$, т.е. $J(P) A_{1} \neq A_{1}$. Ввиду теоремы $2 A_{1}=P z$-главный левый $P$-идеал. Так как $J(P) z \subseteq A_{2} \subset P z$ и между $J(P) z$ и $P z$ нет левых $P$-идеалов, то $A_{2}=J(P) z$.

Б. В силу утверждения А можно считать, что $I A_{1} \supset I A_{2}$. Тогда, пользуясь правосторонним аналогом утверждения А ( $I A_{1}$ и $I A_{2}$ вместо $A_{1}$ и $A_{2}$ и $I^{-1}$ вместо $I$; учесть также равенство $O_{l}\left(I^{-1}\right)=P$ ) получаем: $I A_{1}=b P, I A_{2}=b J(P)$ для некоторого $b \in G$. Тогда $b^{-1} I A_{1}=P$, поэтому $b^{-1} I$ - главный правый $P$-идеал (теорема 2). Пусть $b^{-1} I=z^{-1} P$ для подходящего элемента $z \in G$. Имеем: $A_{1}=z P$ и $A_{2}=z J(P)$.

Продолжим доказательство теоремы. Пусть $J \in \operatorname{spec} P$. Докажем, что $B=$ $I J I^{-1} \in \operatorname{spec} S$. Во-первых, $B$ - собственный идеал конуса $S$, иначе $I J I^{-1}=S$, откуда $S \supseteq I I^{-1} \supseteq I J I^{-1}=S$, т.е. $I I^{-1}=S$. Тогда $I$ - главный правый $P$-идеал (теорема 2), что противоречит предположению о $I$. Во-вторых, если $J=J(P)$, то $B=I J(P) I^{-1}=I I^{-1}=J(S)$, и первичность $B$ доказана. Считаем далее, что $J \neq J(P)$. Тогда, в частности, $J=J(P) J J(P)$ и $J \neq J(P) z, J \neq z J(P)$ ни для какого $z \in G$. Пусть $C$ - идеал конуса $S$, строго содержащий $B$; тогда $I^{-1} C I \supset J=I^{-1} I J I^{-1} I$ (лемма 4). В силу первичности $J$ имеем: $I^{-1} C I I^{-1} C I \supset J$, следовательно, $I^{-1} C^{2} I \supset J$; поэтому $C^{2} \supseteq I\left(I^{-1} C^{2} I\right) I^{-1} \supset I J I^{-1}=B$ (лемма 4, п. Б). Так как множество идеалов конуса линейно упорядочено по включению, то доказано, что $B \in \operatorname{spec} S$.

Аналогично доказьвается первичность идеала $I^{-1} B I$ конуса $P$, если $B \in \operatorname{spec} S$. 
Предположим теперь, что $J \in \overline{\operatorname{spec}} P$. Как и выше, можно считать, что $J \neq$ $J(P)$ и, напоминаем, $I^{-1} I=J(P)$. Из этого равенства и строгого включения $J \subset J(P)$ вытекает, что $P \supset g I \supset J$ для некоторого $g \in I^{-1}$. Так как правые и левые идеалы конуса $S$ линейно упорядочены по включению, то для доказательства вполне первичности идеала $B=I J I^{-1}$ достаточно установить, что $s \in B$, как только $s^{2} \in B$ и $s \in S$. Во-первых, докажем, что $g s g^{-1} \in P$ в этом случае. Действительно, иначе имели бы

$$
J \supseteq g I J \supseteq g I J I^{-1} I=g B I \supseteq g s^{2} I=g s^{2} g^{-1} g I \supseteq g s^{2} g^{-1} J \supseteq J,
$$

ибо $g s^{2} g^{-1} \notin P$. Тогда $J=g s^{2} I$ и $P=O_{r}(I)=O_{r}\left(g s^{2} I\right)=O_{r}(J)$. Но конус $O_{r}(J)$ строго содержит $P$, так как $J$ - вполне первичньй идеал, не совпадающий с $J(P)$ (теорема 1). Полученное противоречие показывает, что на самом деле $g s g^{-1} \in P$. Во-вторых, из соотношения

$$
\left(g s^{2} g^{-1}\right) g I=g s^{2} I \subseteq g I J=J
$$

и вполне первичности идеала $J$ следует, что $g s g^{-1} \in J$. Отсюда получаем: $g s I=$ $g s g^{-1} g I \subseteq J \cdot P=J=g I \cdot J$; из чего вытекает $s J(S)=s I I^{-1} \subseteq I J I^{-1}=B$, и поэтому $s \in B$ в силу первичности $B$ и неравенства $B \neq J(S)$.

Аналогично доказьвается вполнепервичность идеала $I^{-1} B I$ конуса $P$ при условии, что $B \in \overline{\operatorname{spec}} S$.

\section{§2. Конусы с одним вполне первичным идеалом}

В этом параграфе снова $P$ - конус в группе $G$.

Лемма 5. Пусть $I$ - собственный идеал конуса $P$.

A. $Е с л и I^{2}=I$, то $I$ - вполне первичный идеал.

Б. Если пересечение $J=\bigcap_{m=1}^{\infty} I^{m}$ не пусто, то $J$ - вполне первичный идеал.

ДокАЗАтЕльство. А. Достаточно доказать равенство $a I=I$ для всех $a \in$ $P \backslash I$. Доказываем это методом от противного; пусть $a I \subset I$ для некоторого $a \in P \backslash I$. Тогда $P \supset a^{-1} I \supset I$ и $a^{-1} I=a^{-1} I^{2}=\left(a^{-1} I\right) I \subseteq I$ - противоречивые включения.

Б. Если $I^{n}=I^{n+1}$ для некоторого натурального $n$, то $J=I^{n}=I^{2 n}=J^{2}$ и утверждение следует из А. В противном случае для любых $a, b \in P \backslash J$ найдется натуральное число $m$ такое, что $a P \cap b P \supseteq I^{m}$. Тогда $a b P \supseteq a I^{m}=a P I^{m} \supseteq$ $I^{2 m} \neq J$ и, следовательно, $a b \notin J$.

Предположим теперь, что конус $P$ имеет лишь один вполне первичный идеал, который, следовательно, совпадает с радикалом $J(P)$. Опишем структуру моноида $P$-идеалов. Заметим прежде всего, что конус $P$ не имеет собственных надконусов и поэтому, в частности, $O_{r}(I)=P$ для любого правого $P$-идеала $I$.

Случай 1. Предположим, что $J(P)$ - главный правый $P$-идеал, порожденный элементом $z$. Тогда $J(P)=z(P) \supseteq P z$ и $z P z^{-1} \supseteq P$. Отсюда вытекает, что $z P z^{-1}=P$ и $z P=P z$. Согласно лемме 5 получаем, что множество $\bigcap_{n=1}^{\infty} z^{n} P=$ 
$\bigcap_{n=1}^{\infty}(z P)^{n}$ пусто. Так как между $P$ и $z P$ не может быть односторонних идеалов, то это же верно и для "отрезка" $\left[z^{n} P, z^{n+1} P\right]$. Тогда множество односторонних $P$-идеалов совпадает с множеством $\left\{z^{m} P \mid m \in \mathbb{Z}\right\}$ и образует бесконечную циклическую группу по умножению.

Назовем конус $P$ в этом случае дискретным.

Случай 2. Предположим, что радикал $J(P)$ не является главным правым идеалом и не равен $P a P$ ни для какого элемента $a \in J(P)$.

Для произвольно выбранного элемента $a \in J(P)$ обозначим $I=P a P$. Если $I=I J(P)$, то $a=p a b$ для некоторых $p \in P$ и $b \in J(P)$. Так как $P b P \neq J(P)$, то $P b P \subseteq P z \subseteq J(P)$ для подходящего элемента $z \in J(P)$. Из равенства $a=p a b$ следует соотношение $I=I P b P \subseteq I z \subseteq I J(P)=I$, т.е. $I=I z$ и $z^{-1} \in O_{r}(I)=P$, что противоречит принадлежности $z \in J(P)$. Противоречие показьвает, что на самом деле $I \neq I J(P)$. Следовательно, $P a P=d P$ - главный правый $P$-идеал (теорема 2). Так как $d P \supseteq P d$, то $d P d^{-1} \supseteq P$, откуда $d P d^{-1}=P$ и $d P=P d$. Это значит, что соотношение $P a P=d P$ мы можем переписать таким образом: $P d^{-1} a P=P$. Отсюда следует, что $d^{-1} a \in U(P)$, т.е. $d^{-1} a P=P$ и тем самым $a P=d P$ - двусторонний идеал. Как и ранее отсюда вытекает, что $a P=P a$. Следовательно, $g P=P g$ для любого элемента $g \in G$. Ввиду леммы $5 \bigcap_{n=1}^{\infty} a^{n} P=\varnothing$ для любого $a \in J(P)$. Это значит, что линейно упорядоченная отношением включения группа главных $P$-идеалов архимедова, в частности, коммутативна.

Назовем конус $P$ в случае 2 архимедовым.

Случай 3. Предположим, что $J(P)=P a P$ для некоторого $a \in J(P)$, причем $J(P)$ не является главным правым идеалом, а $P$ не содержит собственных идеалов, кроме $J(P)$.

Назовем конус $P$ в этом случае почти простылм (соответствующий пример см. в $\S 4)$.

Случай 4. Предположим, что $J(P)=P a P$ для некоторого элемента $a \in J(P)$, причем $J(P)$ не является главным правым идеалом, и конус $P$ содержит собственньй идеал, отличный от $J(P)$.

Пусть $I$ - идеал конуса $P$, строго содержащийся в радикале $J(P)$. Тогда $I \subseteq$ $a P$, ибо иначе $I \supseteq a P$, откуда $I \supseteq P a P=J(P)$. Будем считать, что $I-$ наибольший идеал, содержащийся в правом идеале $a P$ (применяем лемму Цорна). Докажем, что $I$ - первичный идеал. Действительно, если $b$ и $d$ взяты из $J(P) \backslash I$, то $P b P=P d P=J(P)$, откуда $P b P d P=J(P)^{2}=J(P)$. Последнее равенство имеет место, так как $J(P)$ не является главньм правым идеалом. Итак, $b P d \nsubseteq I$ и первичность идеала $I$ доказана. Из леммы 5 вытекает, что $\bigcap_{n=1}^{\infty} I^{n}=\varnothing$. Так как между $J(P)$ и $I$ нет идеалов, то не будет их и между $I^{m} J(P)$ и $I^{m+1}$ для любого натурального $m$. Все идеалы конуса $P$ перечислены в следуюшей цепочке:

$$
P \supset J(P) \supset I \supseteq I J(P) \supset I^{2} \supseteq I^{2} J(P) \supset \cdots \supset I^{n} \supseteq I^{n} J(P) \supset \cdots .
$$

Нестрогие включения могут быть строгими и тем самым $I$ может быть главным правым (левым) идеалом, а могут и не быть строгими (примеры см. в §4).

Назовем конус $P$ в случае 4 специальным. 
ТеОРема 4. Любой конус $P$ с единственным вполне первичным идеалом является либо архимедовым, либо почти простым, либо специальным. Все три случая реализуются в действительности.

Снова перейдем к произвольному конусу $P$ группы $G$. Рассмотрим два вполне первичных идеала $J_{1}$ и $J_{2}$ конуса $P$ таких, что $J_{1} \supset J_{2}$ и между $J_{1}$ и $J_{2}$ нет других вполне первичных идеалов. В этом случае пару $J_{1}, J_{2}$ будем называть скачком. Пусть $Q_{1}$ и $Q_{2}$ - локализации конуса $P$ по идеалам $J_{1}$ и $J_{2}$, соответственно. Тогда $Q_{2} \supset Q_{1}$ и множество $Q_{12}=Q_{1} \cap U\left(Q_{2}\right)$ будет конусом в группе $U\left(Q_{2}\right)$ с единственным вполне первичньп идеалом $J\left(Q_{12}\right)=J_{1} \cap U\left(Q_{2}\right)$. Действительно, если $T$ - надконус конуса $Q_{12}$ в $U\left(Q_{12}\right)$, то $T_{0}=T \cup J_{2}$ - надконус конуса $P$ в $G$, лежащий строго между $Q_{1}$ и $Q_{2}$. Назовем скачок $J_{1}, J_{2}$ архимедовым (дискретным, почти простым, специальным ), если таков конус $Q_{12}$ в $U\left(Q_{2}\right)$. Следующее предложение усиливает и развивает теорему 3.

ПРЕДЛОЖЕНИЕ 5. В условиях и обозначениях теоремы 3 , если $J_{1}, J_{2}$ - архимедов (дискретный, почти простой, специальный) скачок конуса $P$, то $I J_{1} I^{-1}, I J_{2} I^{-1}$ - скачок конуса $S$ того же типа. Верно и обратное утвержсдение.

ДокАЗАТЕЛЬСТво. - прямое следствие леммы 4, п. Б и теоремы 3. Например, если $J_{1}, J_{2}$ - дискретный скачок, то $J_{1}^{2} \neq J_{1}$ и $\bigcap_{n=1}^{\infty} J_{1}^{n}=J_{2}$. Из леммы 4 вытекает, что тогда $B_{1}^{2} \neq B_{1}\left(B_{i}=I J_{i} I^{-1}, i=1,2\right)$, и так как $\bigcap_{n=1}^{\infty} B_{1}^{n} \in \overline{\operatorname{spec}} S$ ввиду леммы 5 , то $\bigcap_{n=1}^{\infty} B_{1}^{n}=B_{2}$ по теореме 3 . Отсюда следует, что скачок $B_{1}, B_{2}$ дискретный.

\section{§ 3. Автоморфизмы линейно упоря доченных множеств}

В этом параграфе всюду далее $(M, \leqslant)$ - линейно упорядоченное множество (л.у. множество), $G$ - подгруппа группы автоморфизмов Aut $M$ этого л.у. множества.

Пусть $\left\{m_{i} \mid 0 \leqslant i<\lambda\right\}$ - трансфинитное перечисление элементов множества $M$. Это значит, что $i$ и $\lambda$ - ординалы, $m_{i} \neq m_{j}$, если $i \neq j$, и объединение всех $m_{i}$ совпадает с $M$. Для $g \in G$ через $v(g)$ обозначим наименшший индекс $i, i<\lambda$, такой, что $g\left(m_{i}\right) \neq m_{i}$. Если $g$ не является тождественным отображением, то такой индекс сушествует. Для $g=1$ полагаем по определению $v(g)=\lambda$. Нетрудно видеть, что

$$
\begin{gathered}
v(g h) \geqslant \min \{v(g), v(h)\}, \\
v\left(g^{-1}\right)=v(g)
\end{gathered}
$$

для любых $g, h \in G$. Для всякого индекса $\nu, 0 \leqslant \nu \leqslant \lambda$, определим следующие подмножества в $G$ :

$$
\begin{aligned}
P_{\nu} & =\left\{g \in G \mid \text { если } v(g)<\nu, \text { то } g\left(m_{v(g)}\right)>m_{v(g)}\right\}, \\
J_{\nu} & =\left\{g \in P_{\nu} \mid v(g)<\nu\right\}, \\
S_{\nu} & =\left\{g \in P_{\nu} \mid v(g) \geqslant \nu\right\} .
\end{aligned}
$$


Следующая теорема обобщает известный факт теории правоупорядоченных групп (см. [2, глава V, §4, теорема 1]).

Теорема 5 (конусы группы автоморфизмов л.у. множества).

А. Для любого ординала $\nu, 0 \leqslant \nu \leqslant \lambda$, подмножсество $P_{\nu}$ будет конусом c группой обратимых әлементов $S_{\nu}$, и в случае $P_{\nu} \neq G-$ с радикалом $J_{\nu}$. Для $\nu=0$ имеем: $P_{0}=G u J_{0}=\varnothing$. Для $\nu=\lambda P_{\lambda}-$ основной конус; в частности, группа $G$ правоупорядочиваема.

Б. Ecлu $0 \leqslant \nu \leqslant \mu \leqslant \lambda$, mo $P_{\mu} \subseteq P_{\nu} u J_{\mu} \supseteq J_{\nu}$.

В. Если для некоторого ординала $\nu, 0 \leqslant \nu \leqslant \lambda$, подмножсество $\left\{\left\{m_{i} \mid\right.\right.$ $i<\nu\}$ множсества $M$ плотно в $M$, то $P_{\nu}=P_{\lambda}$.

ДокаЗАтельство. А. Заметим, что $P_{\nu}=J_{\nu} \cup S_{\nu}$ и $J_{\nu} \cap S_{\nu}=\varnothing$. Предположим, что $g, h \in P_{\nu}$ и обозначим $i=v(h), j=v(g)$. Если $i<\nu$ и $i \leqslant j$, то $h\left(m_{i}\right) \geqslant i$, откуда $g h\left(m_{i}\right)>g\left(m_{i}\right) \geqslant m_{i}$. В силу (5) имеем $v(g h) \geqslant i$, значит, $g h \in J_{\nu}$. Если $j<\nu$ и $j \leqslant i$, то $h\left(m_{j}\right) \geqslant m_{j}$, откуда $g h\left(m_{j}\right) \geqslant g\left(m_{j}\right)>m_{j}$, и снова $g h \in J_{\nu}$. Если же $i \geqslant \nu, j \geqslant \nu$, то $v(g h) \geqslant \nu$ (см. (5)), следовательно, $g h \in S_{\nu}$.

Тем самьм доказано, что $P_{\nu}$ - полугруппа, $J_{\nu}$ - идеал этой полугруппы, а $S_{\nu}$ - подполугруппа в $P_{\nu}$. Ввиду (6) $S_{\nu}$ будет подгруппой в $P_{\nu}$. Это значит, что $J_{\nu}-$ максимальный правьй (левый) идеал в $P_{\nu}$.

Пусть $g \in G \backslash P_{\nu}$. Тогда $v(g)<\nu$ и $g\left(m_{v(g)}\right)<m_{v(g)}$. Отсюда $m_{v(g)}<$ $g^{-1}\left(m_{v(g)}\right)$. Так как $v\left(g^{-1}\right)=v(g)<\nu$, то $g^{-1} \in J_{\nu}$. Тем самьм $P_{\nu} \cup P_{\nu}^{-1}=G$ и первое утверждение доказано. Равенства $P_{0}=G, J_{0}=\varnothing, S_{\lambda}=\{1\}$, а также утверждение Б следуют сразу из определения множеств $P_{\nu}, J_{\nu}, S_{\nu}$.

В. Пусть $g \in P_{\nu}$. Если $g \in J_{\nu}$, то $g \in J_{\nu} \subseteq J_{\lambda} \subseteq P_{\lambda}$, откуда $g \in P_{\lambda}$. В противном случае $g \in S_{\nu}$. Выберем трансфинитную последовательность $m_{i_{1}}, m_{i_{2}}, \ldots$ такую, что все $i_{j}<\nu$ и $\lim _{j} m_{i_{j}}=m$. Здесь $m$ - любой наперед заданньй элемент. Тогда

$$
g(m)=g\left(\lim _{j} m_{i_{j}}\right)=\lim _{j} g\left(m_{i_{j}}\right)=\lim _{j} m_{i_{j}}=m,
$$

откуда снова $g=1 \in P_{\lambda}$.

Справедливо в некотором смысле обратное утверждение к утверждению теоремы 5. Пусть $P$ - конус в абстрактной группе $G$. Множество $M=\{g P \mid g \in G\}$ линейно упорядочим, считая $g_{1} P \leqslant g_{2} P$ тогда и только тогда, когда $g_{1} P \supseteq g_{2} P$. Элемент $h \in G$ действует слева на $M$ по правилу $g P \rightarrow h g P$. Мы получаем гомоморфизм $\varphi: G \rightarrow$ Aut $M$, причем, если $P$ - основной конус, то $\varphi-$ инъекция и $P=P_{1}$ для $m_{0}=P$ (элементы $m_{1}, m_{2}, \ldots$ из перечисления множества $M$ уже не добавляют никаких новых конусов). Отсюда следует, что любая счетная правоупорядоченная группа может быть вложена в правоупорядоченную группу $\operatorname{Aut}(\mathbb{Q}, \leqslant)$.

Теперь заметим, что любой автоморфизм л.у. множества $M$ может быть единственным образом продолжен до автоморфизма дедекиндова замыкания множества $M$. Ввиду этого будем считать, что л.у. множество $M$ замкнуто в смысле Дедекинда. Далее, если $m \in M-$ наибольший или наименьший элемент множества $M$, то сушествует естественный изоморфизм $\operatorname{Aut}(M) \rightarrow \operatorname{Aut}(M \backslash\{m\})$. Поэтому 
будем считать в дальнейшем, что $M$ не имеет наименьшего и наибольшего элементов. Для всякого элемента $m \in M$ через $\operatorname{St}(m)$ будем обозначать стабилизатор элемента $m$ в группе $G$, т.е. множество всех элементов $g \in G$ таких, что $g(m)=m$. Кроме того, через $P(m)$ будем обозначать конус $\{g \in G \mid g(m) \geqslant m\}$ группы $G$. Ясно, что $\operatorname{St}(m)$ - подгруппа в $G$ и

$$
\begin{aligned}
\operatorname{St}(h(m)) & =h \operatorname{St}(m) h^{-1} \\
P(h(m)) & =h P(m) h^{-1} \\
U(P(m)) & =\operatorname{St}(m)
\end{aligned}
$$

для любого элемента $h \in G$.

Правый $P$-идеал $I$ ( $P$ - конус в абстрактной группе) назовем дивизориальнылм, если $I$ совпадает с пересечением главных правых $P$-идеалов, его содержащих; само это пересечение назовем дивизориальным замыканием и обозначим $\widehat{I}$. Если $I \neq \widehat{I}$, то выберем любой элемент $c$ из дополнения $\widehat{I} \backslash I$. Тогда $\widehat{I} \supseteq c P \supset I$ и между $c P$ и $I$ нет правых $P$-идеалов. Следовательно, $\widehat{I}=c P$ и $I=c J(P)$, причем $J(P)$ не является главным правым $P$-идеалом. Итак, доказано

ПРЕДЛОЖЕНИЕ 6. Если $I$ - не дивизориальный правый $P$-идеал, то $I=$ $c J(P), u J(P)$ не является главным правым $P$-идеалом. В этом случае $\widehat{I}=c P$.

ТЕОРема 6. Пусть әлемент $m \in M$ таков, что $M$ является дедекиндовым замыканием для $G$-орбиты элемента т. Обозначим $P=P(m)$. Сущест-

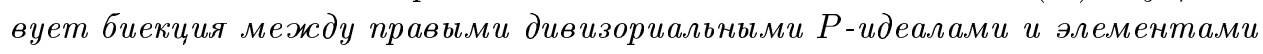
множества $M$, задаваемая правилами:

$$
\begin{gathered}
I-\text { правый } P-\text {-деал } \rightarrow d(I)=\inf \{g(m) \mid g \in I\} \\
a \in M \rightarrow L(a)=\{g \in G \mid g(m) \geqslant a\}
\end{gathered}
$$

Правый $Р$-идеал $L($ a) будет двусторонним $P$-идеалом в том и только том случае, если $g(a) \geqslant а$ для всех $g \in P \quad$ (т.е. из неравенства $g(m) \geqslant m$ следует неравенство $g(a) \geqslant a)$. Далее, $L(a)$ будет вполне первичным идеалом тогда и только тогда, когда $a>m u g(a)=а$ для всех $g \in P$ maких, что $g(m)<a$.

ДокАЗАтЕльство. Монотонность отображений $I \rightarrow d(I)$ и $a \rightarrow L(a)$ ясна. Если $I$ - правый $P$-идеал, то $g_{0} P \supseteq I$ для некоторого $g_{0} \in G$. Тогда для любого $g \in I$ найдется элемент $p \in P$ такой, что $g=g_{0} p$, откуда $g(m)=g_{0} p(m) \geqslant g_{0}(m)$. Следовательно, множество $\{g(m) \mid g \in I\}$ ограничено снизу, и в силу замкнутости по Дедекинду множества $(M, \leqslant)$ элемент $d(I) \in M$ сушествует. Докажем теперь, что $L(a)$ - правый $P$-идеал. Пусть $g \in L(a)$ и $h \in P$. Тогда $g h(m) \geqslant g(m) \geqslant m$, откуда $g h \in L(a)$. Кроме того, $L(a) \neq \varnothing$, так как $M$ не имеет наибольшего элемента, а $G$-орбита элемента $m$ плотна в $M$. Так как $M$ не имеет наименьшего элемента, то найдется элемент $g_{0} \in G$ с условием $g_{0}(m) \leqslant a$. Нетрудно видеть, что $g_{0} P \supseteq L(a)$ в этом случае. Действительно, если $h \in L(a)$, то $g_{0}^{-1} h(m) \geqslant$ $g_{0}^{-1}(a) \geqslant m$, откуда $g_{0}^{-1} h \in P$. Итак, доказано, что $L(a)-$ правьй $P$-идеал. Если $L(a)$ не дивизориален, то $L(a)=h J(P)$ согласно предложению 5 , причем $J(P)$ 
не является главным правым $P$-идеалом. Следовательно, $m$, а значит, и $h(m)$ не являются изолированной точкой. Тогда $h(m) \geqslant a$, ибо иначе $h p(m)<a$ для некоторого элемента $p \in J(P)$, что противоречит равенству $L(a)=h J(P)$. Итак, $h(m) \geqslant a$ и $h \in L(a)=h J(P),-$ снова противоречие. Противоречие показьвает, что правьй идеал $L(a)$ является дивизориальным.

Докажем, что $L d(I)=\widehat{I}$. Во-первых, $I \subseteq L d(I)$. Во-вторых, если $h \in G \backslash I$, то $h P \supseteq L d(I)$, ибо для любого элемента $g \in L d(I)$ имеет место неравенство $g(m) \geqslant$ $d(I)$, из которого следует, что $h^{-1} g(m) \geqslant h^{-1} d(I) \geqslant m$. Тем самым доказано, что $L d(I)=\widehat{I}$. Докажем равенство $d L(a)=a$. Неравенство $d L(a) \geqslant a$ очевидно. Предположим, что $d L(a)>a$. Так как $M$ - дедекиндово замыкание для $G$-орбиты элемента $m$, то найдется автоморфизм $g \in G$ такой, что $a \leqslant g(m)<d L(a)$. Тогда $g \in L(a)$ и $g(m)<\inf \{h(m) \mid h \in L(a)\} \leqslant g(m)$. Это противоречие показьвает, что на самом деле $a=d L(a)$.

Итак, первое утверждение теоремы доказано. Предположим теперь, что $L(a)-$ $P$-идеал, но $g(a)<a$ для некоторого элемента $g \in P$. Еще раз пользуясь тем, что $M$ - дедекиндово замькание $G$-орбиты элемента $m$, найдем такой автоморфизм $h \in G$, что $g^{-1}(a)>h(m) \geqslant a$. Тогда $a>g h(m)$ и $h \in L(a)$. Следовательно, $g h \in L(a)$ ввиду того, что $L(a)-P$-идеал, из чего следует неравенство $g h(m) \geqslant$ a. Это неравенство противоречит предыдущему. Противоречие показывает, что $g(a) \geqslant a$ для любого $g \in P$.

Наоборот, пусть $g(a) \geqslant a$ для всех $g \in P$ и $h \in L(a)$ - произвольный элемент. Тогда $g h(m) \geqslant g(a) \geqslant a$, откуда $g h \in L(a)$, и, значит, $L(a)-$ двусторонний Р-идеал.

Докажем последнее утверждение теоремы. Пусть $L(a)$ - вполне первичный идеал. Тогда неравенство $a>m$ следует из того, что $L(a) \subset P$. Пусть $g \in P$ и $g(m)<a$. В силу предыдушего утверждения, равенство $g(a)=a$ следует из невозможности строгого неравенства $g(a)>a$. Если бы $g(a)>a$, то нашелся бы элемент $h \in G$ такой, что $g(a)>h(m) \geqslant a$. Тогда $g^{-1} h(m) \geqslant g^{-1}(a)>m$, откуда $g^{-1} h \in P$. Но одновременно, $g^{-1} h(m)<a$, что означает $g^{-1} h \notin L(a)$. Итак, $g$ и $g^{-1} h$ не принадлежит $L(a)$, но $h=g \cdot g^{-1} h \in L(a)$. Это противоречие с вполне первичностью идеала $L(a)$.

Обратно, пусть условие $g(a)=a$ вьполнено для всех $g \in P$ таких, что $g(m)<a$ и $a>m$. Тогда в силу второго утверждения теоремы $L(a)$ - идеал конуса $P$. Если $g, h \in P \backslash L(a)$, то $g(m)<g(a)=a$ и $h(m)<h(a)=a$. Следовательно, $g h(m)<g h(a)=a$, откуда $g h \notin L(a)$. Это доказьвает, что $L(a) \in \overline{\operatorname{spec}} P$.

Доказательство теоремы завершено.

Отрезок $[a, b] \subseteq M$ назовем сжимаемым (более точно: $G$-сжимаемьм), если существует автоморфизм $g \in G$ такой, что

$$
a<g(a)<g(b)<b
$$

СлЕДСТВИЕ. В условиях и обозначениях теоремы 6 конус $P$ будет почти простым тогда и только тогда, когда любой отрезок $[m, g(m)]$, где $g \in J(P)$, сжимаем. Конус $P$ будет архимедовым, если и только если $\lim _{n \rightarrow+\infty} g^{n}(m)=+\infty$ для любого әлемента $g \in J(P)$. Конус $P$ будет дискретныц в том и только том случае, когда $(M, \leqslant) \simeq(\mathbb{Z}, \leqslant)$ и $G \simeq(\mathbb{Z},+)$. 
ДокАЗАТЕЛьство. Первое утверждение следует из теоремы 6 , а второе - из теоремы 4.

Предположим, что конус $P$ дискретен, и $z$ - порождающий элемент радикала этого конуса. Обозначим $m_{i}=z^{i}(m)(i \in \mathbb{Z})$. Если $u \in U(P)$, то $u(m)=m$. Для любого целого числа $i$ найдется элемент $u_{i} \in U(P)$ такой, что $u z^{i}=z^{i} u_{i}$. Тогда $u\left(m_{i}\right)=u z^{i}(m)=z^{i} u_{i}(m)=z^{i}(m)=m_{i}$. Следовательно, объединение всех $m_{i}$ будет $G$-орбитой элемента $m$. Это объединение замкнуто по Дедекинду, откуда $M=\cup\left\{m_{i}\right\} \simeq(\mathbb{Z}, \leqslant)$. Тогда $U(P)=\{1\}$ и, значит, $G=\operatorname{gr}\{z\} \simeq(\mathbb{Z},+)$.

\section{§4. Примеры}

Пусть $E$ - евклидова плоскость с ортонормированным базисом $e_{1}, e_{2} ; S$ - окружность радиуса 1 с центром в начале координат. Параметризуем $S$ полуинтервалом $[0,2 \pi)$, сопоставляя числу $t \in \mathbb{R}$ конец единичного вектора $e(t)=e_{1} \cos t+e_{2} \sin t$. Наоборот, если $a$ - произвольный ненулевой вектор пространства $E$, то через $\arg a$ обозначим то единственное число $t \in[0,2 \pi)$, для которого $e(t)=a /\|a\|$. Группу линейных автоморфизмов пространства $E$ будем отождествлять с $\mathrm{GL}(2, \mathbb{R})$, отождествление строится по базису $e_{1}, e_{2}$. Если $s \in \mathrm{GL}(2, \mathbb{R})$, то через $s(t)$ будем обозначать аргумент вектора $s(e(t))$; тем самьм задано действие $\mathrm{GL}(2, \mathbb{R})$ на $S$, ядром которого служат гомотетии. Рассмотрим прямую $\mathbb{R}$ как универсальное накрытие окружности $S$, эпиморфизм $\mathbb{R} \rightarrow[0,2 \pi)$ задается таким образом: $t \rightarrow \arg e(t)$. Пусть $G$ - универсальная накрывающая группа группы $\operatorname{SL}(2, \mathbb{R})$. Известно, что центр $G$ - бесконечная циклическая группа, порожденная элементом $z$ таким, что $z^{2}$ порождает ядро накрытия $\tau: G \rightarrow \mathrm{SL}(2, \mathbb{R})$, причем $\tau(z)=-1_{E}$. Заметим, что группа $\mathrm{SL}(2, \mathbb{R})$ действует на полуинтервале $[0,2 \pi)$ локально монотонно. Поднимем действие группы $\mathrm{SL}(2, \mathbb{R})$ на $[0,2 \pi)$ до монотонного действия $G$ на $\mathbb{R}$ так, что $z$ - сдвиг множества $\mathbb{R}$ на число $\pi$ и диаграмма

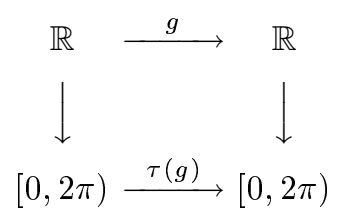

коммутативна для всякого элемента $g \in G$. Для элемента $s \in \operatorname{SL}(2, \mathbb{R})$ через $s_{0}$ будем обозначать то единственное монотонное преобразование $g \in G$, для которого $\tau(g)=s$ и $g(0)=s(0)$. Тогда прообраз элемента $s$ при эпиморфизме $\tau$ совпадает с множеством $s_{0} z^{2 i}(i \in \mathbb{Z})$. Более подробно об универсальной накрывающей группе $G$ см., например, в [6, лекция 20].

Лемма 6. Пусть $a_{1}, a_{2}, a_{3}, b_{1}, b_{2}, b_{3}$ - действительнье числа из полуинтервала $[0, \pi)$ такие, что $a_{1}<a_{2}<a_{3} u b_{1}<b_{2}<b_{3}$. Тогда существует әлемент $s \in \mathrm{SL}(2, \mathbb{R})$ такой, что $s\left(a_{i}\right)=b_{i}$ для $i=1,2,3$.

ДоКАЗАТЕЛЬСТВо достаточно провести для случая $a_{1}=0, a_{2}=\pi / 4, a_{3}=\pi / 2$. Матрица $\widetilde{s}=\left(\begin{array}{cc}\cos b_{1} & \cos b_{3} \\ \sin b_{1} & \sin b_{3}\end{array}\right)$ очевидно удовлетворяет условиям $\widetilde{s}\left(a_{1}\right)=b_{1}$, $\widetilde{s}\left(a_{3}\right)=b_{3}$, и определитель ее $d=\sin \left(b_{3}-b_{1}\right)$ больше нуля. Остается подобрать положительное число $\alpha$ так, чтобы элемент $s=\left(\begin{array}{cc}\alpha \cos b_{1} & \frac{\cos b_{3}}{\alpha d} \\ \alpha \sin b_{1} & \frac{\sin b_{3}}{\alpha d}\end{array}\right)$ групшы $\operatorname{SL}(2, \mathbb{R})$ 
удовлетворял условию $s\left(a_{2}\right)=b_{2}$ (двум другим он заведомо удовлетворяет). Так как $s\left(a_{2}\right) \rightarrow b_{1}$, если $\alpha \rightarrow+\infty$, и $s\left(a_{2}\right) \rightarrow b_{3}$, если $\alpha \rightarrow 0+0$, то такое число $\alpha$ существует в силу теоремы Коши о промежуточном значении.

Теорема 7. Конус $P=\{g \in G \mid g(0) \geqslant 0\}$ группы $G$ специален, $z P$ первичный, но не вполне первичный идеал конуса $P$. Группа $T_{0}$ обратимым әлементом конуса $P$ посредством $\tau$ изоморфно отображается на группу верхнетреугольных матрии из $\mathrm{SL}(2, \mathbb{R})$ с положительными әлементами на главной диагонали.

ДокаЗАтельство. Ясно, что $z \in J(P)$ и $z P=P z$-идеал конуса $P$. Далее, для любого элемента $g \in G$ найдется натуральное число $n$ такое, что $z^{n}(0)>g(0)$, откуда следует, что $\bigcap_{n=1}^{\infty} z^{n} P=\varnothing$. Из леммы 6 и теоремы 6 вытекает, что между $J(P)$ и $z P$ нет идеалов. Следовательно, $J(P)$ и $z P$ - единственные первичные идеалы. Идеал $z P$ не является вполне первичньм, так как в $J(P)$ существуют корни из $z$ любой степени $n$ (сдвиги множества $\mathbb{R}$ на величину $\pi / n, n \in \mathbb{N}$ ).

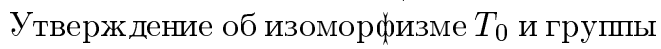

$$
T=\left\{\left(\begin{array}{cc}
a & b \\
0 & 1 / a
\end{array}\right) \mid a, b \in \mathbb{R} ; a>0\right\}
$$

проверяется прямьм вычислением.

Группа $T$ монотонно действует на интервале $(0, \pi)$ (сужение действия $\operatorname{GL}(2, \mathbb{R})$ с $S$ на $(0, \pi))$ и нет нужды поднимать это действие до действия $T_{0}$ на $\mathbb{R}$, ибо интервал $(0, \pi)$ - л.у. множество, замкнутое по Дедекинду и не содержащее наименьшего и наибольшего элементов. Для $\alpha \in(0, \pi)$ введем обозначение $Q(\alpha)=\{s \in T \mid$ $s(\alpha) \geqslant \alpha\}$.

ПРЕДЛОЖЕНИЕ 7. $Q(\alpha)$ - почти простой конус и $U(Q(\alpha)) \simeq\left(\mathbb{R}^{>0}, \cdot\right)$.

ДокАЗАТЕльство. Первое утверждение следует из леммы 6 и следствия теоремњ 6. Справедливость второго утверждения проверяется прямьм вычислением.

Рассмотрим теперь три примера счетных конусов. Первый пример связан с правоупорядоченной группой, построенной Д. М. Смирновым [7]:

$$
\mathrm{ST}=\left\{\left(\begin{array}{ll}
k & a \\
0 & 1
\end{array}\right) \mid a, k \in \mathbb{Q} ; k>0\right\} .
$$

Группа ST так же, как и $T$, действует монотонно на интервале $(0, \pi)$. При отображении $\left(\begin{array}{ll}k & a \\ 0 & 1\end{array}\right) \rightarrow\left(\begin{array}{cc}\sqrt{k} & a / \sqrt{k} \\ 0 & 1 / \sqrt{k}\end{array}\right)$ группа ST изоморфно переходит в плотную подгруппу групшы $T$, сохраняя при этом действие на интервале $(0, \pi)$. Следовательно, $\mathrm{SQ}(\alpha)=\{s \in \mathrm{ST} \mid s(\alpha) \geqslant \alpha\}$ - почти простой конус для любого элемента $\alpha \in(0, \pi)$. Нетрудно подобрать $\alpha$ так, что группа обратимых элементов конуса SQ $(\alpha)$ будет единичной, и тем самым группа ST будет правоупорядоченной. Действительно, если $\varepsilon-$ положительное иррациональное число, то вектор $\left(\begin{array}{ll}k & a \\ 0 & 1\end{array}\right)\left(\begin{array}{l}1 \\ \varepsilon\end{array}\right)=\left(\begin{array}{c}k+a \varepsilon \\ \varepsilon\end{array}\right)$ не может быть коллинеарньпм вектору $e_{1}+\varepsilon e_{2}$; поэтому $\alpha=\arg \left(e_{1}+\varepsilon e_{2}\right)$ - искомое число. В этом случае $\operatorname{SQ}(\alpha)$ - основной почти простой конус (другое доказательство см. в [8]). 
Рассмотрим теперь дискретную подгруппу $\mathrm{SL}(2, \mathbb{Z})$ группы $\mathrm{SL}(2, \mathbb{R})$. Ее универсальная накрываюшая группа $\mathrm{GZ}$ изоморфна прообразу $\mathrm{SL}(2, \mathbb{Z})$ при эпиморфизме $\tau$ и порождается элементами $u, v$ такими, что

$$
\begin{array}{clrl}
u^{3} & =v^{2}=z, & \tau(u) & =\left(\begin{array}{cc}
0 & -1 \\
1 & 1
\end{array}\right), \\
\tau(v) & =\left(\begin{array}{cc}
0 & -1 \\
1 & 0
\end{array}\right), & & u(0)=v(0)=\pi / 2 .
\end{array}
$$

В частности, $v(r)=r+\pi / 2$ для любого $r \in \mathbb{R}$. Более подробно о группе GZ можно узнать в $[9, \S 10]$.

Элементы $A=\left(\begin{array}{ll}1 & 2 \\ 0 & 1\end{array}\right)$ и $B=\left(\begin{array}{ll}1 & 0 \\ 2 & 1\end{array}\right)$ порождают в $\mathrm{SL}(2, \mathbb{Z})$ свободную подгруппу ранга 2 , которая совпадает с группой

$$
F=\left\{\left(\begin{array}{ll}
a & b \\
c & d
\end{array}\right) \in \mathrm{SL}(2, \mathbb{Z}) \mid a \equiv d \equiv 1(\bmod 4), c \equiv b \equiv 0(\bmod 2)\right\}
$$

(см. [9, 14.2.1 и 14.2.2]). Следовательно, элементы $A_{0}, B_{0}$ порождают в $G$ свободную подгруппу $F_{0}$, которая изоморфно отображается на $F$ посредством эпиморфизма $\tau$.

Лемма 7. Для любых чисел $a_{1}, a_{2}$ таких, что $0 \leqslant a_{1}<a_{2}<\pi$ найдется әлемент $g \in F_{0}$, для которого $a_{1}<g\left(a_{1}\right)<g\left(a_{2}\right)<a_{2}$ (т.е. интервал $\left(a_{1}, a_{2}\right)$ - $F_{0}$-сжимаем).

ДокаЗАТЕЛЬство. Во-первых, заметим, что множество $\{s(0) \mid s \in F\}$ всюду плотно на полуинтервале $[0,2 \pi)$, ибо множество рациональных чисел вида $c / a$, где $a, c \in \mathbb{Z}, a \equiv 1(\bmod 4), c \equiv 0(\bmod 2)$ всюду плотно на $\mathbb{R}$. Во-вторых, $A^{n}(\alpha) \rightarrow 0$ при $n \rightarrow+\infty$ для любого $\alpha \in[0, \pi)$. Пусть элемент $s \in F$ таков, что $\beta=s(0) \in\left(a_{1}, a_{2}\right)$. Тогда $s(U) \subseteq\left(a_{1}, a_{2}\right)$ для некоторой достаточно малой правой окрестности $U$ нуля. Выберем натуральное число $n$ такое, что $A^{n}\left(a_{1}\right)$ и $A^{n}\left(a_{2}\right)$ принадлежали бы окрестности $U$. Тогда $g=\left(s A^{n}\right)_{0}$ - искомый элемент.

TЕОРема 8. Для любого действительного числа $\alpha$ множество $\mathrm{PZ}(\alpha)=$ $\{g \in \mathrm{GZ} \mid g(\alpha) \geqslant \alpha\}$ - специальный конус в группе $\mathrm{GZ}$, a $\mathrm{PF}(\alpha)=\left\{g \in F_{0} \mid\right.$ $g(\alpha) \geqslant \alpha\}$ - специальный конус в группе $F_{0}$. В первом случае $z \mathrm{PZ}(\alpha)$ является первичным, но не вполне первичным идеалом, а во втором таковым будет идеал $I=\left\{g \in F_{0} \mid g(\alpha) \geqslant \alpha+\pi\right\}$.

Группы $U(\mathrm{PZ}(\alpha))$ и $U(\mathrm{PF}(\alpha))$ либо единичнье, либо бесконечнье ииклические. Первый случай имеет место, если $\alpha=\arg \left(e_{1}+\varepsilon e_{2}\right)$, әде число $\varepsilon \in \mathbb{R}$ не принадлежит ни одному квадратичному расширению поля $\mathbb{Q} ;$ причем $I$ тогда не будет главным правым (левым. $)$ РF $(\alpha)$-идеалом.

ДокАЗАТЕЛЬСтво. Пусть $\alpha<b_{1}<b_{2}<\alpha+\pi$. В силу плотности $F_{0}$-орбиты нуля на прямой $\mathbb{R}$, найдется элемент $g \in F_{0}$, для которого $h\left(b_{1}\right)<g h\left(b_{1}\right)<$ $g h\left(b_{2}\right)<h\left(b_{2}\right)$. Тогда $b_{1}<h^{-1} g h\left(b_{1}\right)<h^{-1} g h\left(b_{2}\right)<b_{2}$, т.е. интервал $\left(b_{1}, b_{2}\right)$ $F_{0}$-сжимаем. Применяя следствие теоремы 6 так же, как и в доказательстве предложения 7 , получаем, что $\mathrm{PZ}(\alpha)$ и $\mathrm{PF}(\alpha)$ - специальные конусы, а $z \mathrm{PZ}(\alpha)$ и $I$ первичные, но не вполне первичные идеалы. 
Предположим, что $g(\alpha)=\alpha+\pi$ или $g(\alpha)=\alpha$ для некоторого элемента $g \in \mathrm{GZ}$ и $\alpha=\arg \left(e_{1}+\varepsilon e_{2}\right)$. Тогда $e_{1}+\varepsilon e_{2}$ является собственньм вектором матрицы $\tau(g)=$ $\left(\begin{array}{ll}a & b \\ c & d\end{array}\right) \in \mathrm{SL}(2, \mathbb{Z})$ с собственньп числом $\beta$. Отсюда $a+b \varepsilon=\beta$ и $c+d \varepsilon=\beta \varepsilon$, поэтому $b \varepsilon^{2}+(a-d) \varepsilon+c=0$. Если $\varepsilon$ не принадлежит ни одному квадратичному расширению поля $\mathbb{Q}$, то $b=0, a=d$ и $c=0$. Единственно возможные варианты в этом случае $-g=z$ и $g=1$. Заметим, что $z \notin F_{0}$. Это рассуждение показьвает, что $U(\mathrm{PZ}(\alpha))=U(\mathrm{PF}(\alpha))=1$ и $I$ не является главным правым (левым) идеалом при выбранном как и выше $\varepsilon$.

Если $U(\mathrm{PZ}(\alpha)) \neq 1$, то $g(\alpha)=\alpha$ для некоторого не единичного элемента $g \in \mathrm{GZ}$. Обозначим тогда через $H$ образ группы $U(\mathrm{PZ}(\alpha))$ при эпиморфизме $\tau$. Из построения универсальной накрываюшей групшы следует, что отображение $\tau: U(\mathrm{PZ}(\alpha)) \rightarrow H$ будет изоморфизмом групп. Обозначим через $K \mathbb{Q}$-подалгебру, порожденную в алгебре $2 \times 2$-матриц над $\mathbb{Q}$ группой $H$. Так как все матрицы из $H$ имеют совпадающий собственный вектор, то $\operatorname{dim}_{\mathbb{Q}} K \leqslant 3$. Пусть $\operatorname{tg} \alpha \notin \mathbb{Q}$, тогда любой не единичньй элемент $A \in H$ порождает квадратичное расширение $\mathbb{Q}[A]$ поля $\mathbb{Q}$. Следовательно, $\operatorname{dim}_{\mathbb{Q}} K=2$, и поэтому $K$ - поле. Обозначим через $C$ целое замыкание кольца $\mathbb{Z}$ в поле $K$. По теореме Дирихле (см. [10, глава II, $\S 4$, теорема 5]) группа $U(C)$ обратимых элементов кольца $C$ будет прямым произведением циклической бесконечной группы на группу корней из 1 . Так как $H \simeq U(\mathrm{PZ}(\alpha))-$ линейно упорядочиваемая группа и $H \subseteq U(C)$, то $H$ - бесконечная циклическая группа. Если $\operatorname{tg} \alpha \in \mathbb{Q}$, то, изменяя базис, сводим доказательство к случаю $\alpha=0$; тогда $U(\mathrm{PZ}(\alpha))=\operatorname{gr}\left(\begin{array}{ll}1 & 1 \\ 0 & 1\end{array}\right)$.

СлЕДСТВИЕ. В свободной группе ранга 2 существует основной специальнъй конус.

В связи с этим результатом возникает вопрос: сушествует ли в свободной группе основной почти простой конус?

Рассмотрим теперь элементы $x=u^{-1} v u^{-1}$ и $y=v$, порождающие группу GZ и имеющие соотношение $x y^{2} x=y$. В работе [4] доказано, что $P_{x y}=$ $\operatorname{semigr}\{x, y\}$ - основной конус в $\mathrm{GZ}, J=\bigcap_{n=1}^{\infty} x^{n} P_{x y}-$ вполне первичньй идеал, а $I=\bigcup_{n=1}^{\infty} x^{-n} y^{3} P_{x y}$ - первичный, но не вполне первичный идеал. С точки зрения развитой в настоящей работе теории этот факт получает простую геометрическую трактовку. Действительно, можно подсчитать, что $x(-\pi / 4)=-\pi / 4$, тогда конус $\mathrm{PZ}(-\pi / 4)$ содержит $x$ и $y$, причем $U(\mathrm{PZ}(-\pi / 4))=\operatorname{gr}\{x\}$. Следовательно, $\mathrm{PZ}(-\pi / 4)$ - локализация конуса $P_{x y}$ по идеалу $J$.

В заключение отметим без доказательства несложный, но интересный факт о группе $G=\operatorname{Aut}(\mathbb{Q}, \leqslant)$. Пусть $m_{0}, m_{1}, m_{2}, \ldots$ - перечисление рациональных чисел. Тогда

$$
\varnothing=J_{0} \subset J_{1} \subset J_{2} \subset \cdots \subset J_{\omega} \subset P_{\omega} \subset \cdots \subset P_{2} \subset P_{1} \subset P_{0}=G
$$

$\left(J_{i}, J_{i+1}\right)$ - почти простой скачок основного конуса $P_{\omega}$ для любого натурального числа $i \geqslant 0$ (тем самым, $J_{i}, i \geqslant 1$, и $J_{\omega}$ - все собственные идеалы конуса $P_{\omega}$ ), а группа $S_{i}$ изоморфна прямому произведению $i+1$ экземпляров группы Aut $\mathbb{Q}$. 


\section{Список литературы}

1. Дубровин Н. И. Рациональное замькание групповых колец левоупорядоченных групп // Матем. сб. 1993. Т. 183. № 7. С. 3-48.

2. Кокорин А. И., Копьтов В. М. Линейно упорядоченные группы. М.: Наука, 1972.

3. Дубровина T. В. Один пример конечно порожденной правоупорядоченной группы // Тезисы XIX Всесоюзной алгебраической конференции. Львов, 1987.

4. Дубровин Н. И. Пример цепного первичного кольца с нильпотентньми элементами // Матем. сб. 1983. Т. 120 (162). № 3. С. 441-447.

5. Bergman G. M. Right orderable groups that are not locally indicable // Pacific J. Math. 1991. V. 147. P. 243-248.

6. Постников M. М. Лекции по геометрии. Семестр V. Группы и алгебры Ли. М.: Наука, 1982.

7. Смирнов Д. М. Правоупорядоченные группы // Алгебра и логика. 1966. Т. 5. №6. C. $41-59$.

8. Дубровин Н. И. Цепные области // Вестник МГУ. Сер. матем., мех. 1980. № 2. С. 51-54.

9. Каргаполов М.И., Мерзляков Ю. Н. Основы теории групп. М.: Наука, 1982.

10. Боревич З. И., Шафаревич И. Р. Теория чисел. М.: Наука, 1964.

Владимирский политехнический институт

Поступила в редакцию

23.06.1993 